\title{
MÁS DE UN SIGLO PARA PRESENCIAR LA ALFABETIZACIÓN DIGITAL EN EL PERÚ
}

\author{
MORE THAN A CENTURY TO WITNESS DIGITAL LITERACY IN PERU \\ (iD) Reynaldo Sucari León ${ }^{*}$, (iD César Teófilo Zambrano Arce ${ }^{1}$, (D) Yolanda Aroquipa Durán', \\ (iD) Nancy Chambi Condori ${ }^{1}$, (iD) Rebeca Vítor Flores ${ }^{1}$ \\ rsucari@unah.edu.pe; czambrano@unah.edu.pe; yaroquipa@unah.edu.pe; nchambi@unap.edu.pe; \\ 1712810225@unah.edu.pe \\ ${ }^{1}$ Universidad Nacional Autónoma de Huanta, Ayacucho, Perú
}

*Correspondencia: Reynaldo Sucari León. Email: rsucari@unah.edu.pe

Recibido: 27.06.21 | Aprobado: 15.07.21

\section{RESUMEN}

La tecnología digital desde su aparición en el mundo, tomó su tiempo para llegar al Perú en el siglo XX, y lentamente va adquiriendo su consolidación hoy en el siglo XXI gracias a los procesos de alfabetización digital, por ello es necesario y urgente que los peruanos debemos contar con una alfabetización digital sostenible promovida por el sector educación; el Ministerio de Educación y las Universidades, para que profesionales o no se desenvuelvan de manera óptima respeto al manejo de la información en todos los sectores y ámbitos, aportando de esa manera al desarrollo de nuestro país.

Palabras clave: Alfabetización digital, nuevas formas de comunicación, tecnología digital.

\section{ABSTRACT}

Digital technology since its appearance in the world, took its time to reach Peru in the twentieth century, and little by little it is acquiring its consolidation today in the $21 \mathrm{st}$ century thanks to the processes of digital literacy, therefore, it is necessary and urgent that Peruvians have a sustainable digital literacy promoted by the education sector; the Ministry of Education and Universities, for professionals or not to function optimally with respect to the management of information in all sectors and areas, thus contributing to the development of our country.

Keywords: Digital literacy, new forms of communication, digital technology. 


\section{INTRODUCCIÓN}

La presente investigación hace referencia al tema de la alfabetización digital y su transición desde el siglo XX hasta la actualidad (siglo XXI), entendiendo la alfabetización digital como la habilidad que desarrolla una persona para buscar, analizar y comprender la información con el apoyo de la tecnología y los recursos digitales. Así mismo al proceso de alfabetización digital se le conoce con otras denominaciones como alfabetización multimedia o alfabetismo digital.

El propósito fundamental de este estudio es presentar una descripción de la transición en tecnología digital por la cual el Perú está atravesando, enfocando dos tiempos bien marcados el siglo XX por una parte y concluyendo con los sucesos del siglo XXI.

Los subtemas abordados se hallar organizados en dos secciones, la primera bajo la denominación "El Perú en el siglo XX y sus formas de comunicación" tratando temas como: el peruano del siglo XX, inserción en las nuevas formas de comunicación, alfabetización digital como inclusión social, nuevas formas de inserción en la cultura digital, alfabetización digital para una práctica socioeconómica, y nuevas perspectivas de alfabetización digital. La segunda sección enfoca los temas agrupados en el título "Perú en el siglo XXI consolidando la digitalización" tocando tópicos como: el peruano multialfabetizado en el siglo XXI, valores a tomar en cuenta dentro de la alfabetización digital, influencia positiva y negativa del empleo de la tecnología, y prácticas de inserción de la alfabetización digital.

\section{PERÚ EN EL SIGLO XX Y SUS FORMAS DE COMUNICACIÓN}

\section{E1 peruano del siglo XX}

Alvin Tolfer (1993) utiliza el término ola para descifrar como se divide la civilización, pasando de una etapa agrícola (sin presencia de la tecnología digital), a otra industrial y a una tercera ola llamada "era de la información"; donde la invasión de la tecnología digital es desmedida en todos los ámbitos. El siglo XX es una transición entre la segunda y la tercera ola. En ese sentido podemos describir al intelectual de dicha transición histórica, como un hombre que buscaba darle sentido a su vida, la historia debería de tener finalidad para analizar lo que estaba sucediendo. La preocupación del intelectual azotado por las guerras y revoluciones, era la participación de la masa en política, y como éstas se articulaban como actor revolucionario o de progreso. Ya Nietzsche se encargaba de cuestionar la teoría racionalista y la herencia de la ilustración. Así como al conjunto de teorías que justifican que el hombre controle sus emociones, las mismas que responde al diseño de Sócrates y Platón, en el cual la religión crea un rol de propaganda, de represión y de control social a las masas (Fontana, 2005), es decir era una realidad muy diferente a la actual.

De hecho, pasaron muchas etapas como el capitalismo que perfiló al hombre pragmático y utilitarista, todo poderoso, confiado en sus avances, hasta que la guerra lo hace dudar del progreso aparente de la sociedad y de la cultura occidental. Asimismo, lo positivo es que el hombre retoma el humanismo y el debate sociológico, permitiéndole 
un desarrollo increíble de las humanidades, que hoy en pleno siglo XXI aún no es superado (Ayllón, Gutiérrez, \& González, 2008).

A nuestro entender Jean Paul Sarte; intelectual radical que rechaza un premio nobel y que convierte las calles de París en centro de agitación intelectual, plantea la vida como problema y la elección como acto obligatorio, es decir elegimos el cambio o nos quedamos sin progreso. Otro aspecto que se rescata del gran Sarte es que el ateísmo involucra un aspecto ético, predominando la dignidad y lo humano, claro está sin un Dios que castiga a la humanidad, sino guiados por el ideal de convertirnos en auténticos dueños de nuestra vida (Ayllón et al., 2008)

La propaganda radical se dio durante el siglo XX, aturdido por el impulso de la gran revolución industrial, así la administración también tuvo que soportar la carga de tener jefes muy estrictos que no tenían ninguna consideración por sus trabajadores; explotados y descontentos, dado que la industria exigía el menor derroche de recursos con precios bajos y competitivos en los mercados mundiales, esta segunda ola, de la revolución industrial fue muy breve, nuestros abuelos y padres, son el resultado de esa generación, disciplinados, formados para el trabajo industrial, una sociedad conflictiva pero intensa, donde el marxismo profetizaba el fin del capitalismo, que logró convertir a Rusia en una potencia, y el triunfo del modelo socialista era visto con asombro por países de desarrollo capitalista atrasado. (Toffler, 1993)

Sin embargo, en el Perú como en Europa, la disolución de la Unión Soviética ha previsto la culminación de la historia en el cual "la lucha de clases" se extinguía del vocabulario académico, el esquema preconizado en las universidades llegaba a su fin tras el fracaso del modelo de sociedad, el predominio del capitalismo era evidente, el neoliberalismo era la religión del momento, se vivía en un Estado que remataba sus empresas al mejor postor y todo quedaba a la oferta y demanda. Entonces surge una interrogante ¿por qué sobrevivió el Estado Liberal en el siglo XX? La respuesta es que el Estado moderno no surge de la tradición, ni de la fe, sino del resultado del debate político entre los ciudadanos. En otras palabras, las revoluciones y las guerras habían fortalecido el espíritu liberal, además la vigencia de establecer los derechos humanos, así como los derechos de tercera generación o de los pueblos, además de la condena de los crímenes contra la humanidad (Fukuyama, 1992)

En la actualidad en Europa queda China de Mao como un museo del pasado comunista del siglo XX, proyectándose de manera capitalista para el mundo, pero de un Estado de bienestar para su propia gente, donde las brechas sociales disminuyen quizá a pasos lentos, pero disminuye. Aquí en Perú de forma similar se proyecta la sociedad capitalista, aunque con algunas diferencias. Aunque para los ciudadanos actuales interese poco que son libertades, la oferta y la demanda son inherentes a su economía. Pero la sociedad capitalista crece a merced del Estado liberal. A fines del siglo XX la sociedad se caracterizó por ser prosumidor, una sociedad dominada por aquel individuo que diseña lo que desea consumir, este modelo todavía es joven en comparación de lo que es hoy, en estos momentos. (Toffler, 1993) 


\section{INSERCIÓN EN LAS NUEVAS FORMAS DE COMUNICACIÓN}

Hace un siglo las formas de comunicación con que contaban los peruanos no era igual a la que hoy se tiene, ya que la tecnología ha cambiado a pasos agigantados, este proceso y más aún con el empleo de los medios, como por ejemplo desde la aparición de la radio, pasando por el teléfono, la televisión hasta llegar al correo postal, hoy los peruanos contamos con los celulares denominados teléfonos inteligentes, TV-cable, utilizamos el Internet y de manera desmedida empleamos las redes sociales. Es innegable que los cambios tecnológicos que se dieron no solo en el Perú, sino en todos los países durante en las últimas décadas, fueron muy variadas, numerosas y generaron cambios profundos en toda la historia de la humanidad. (Ferrer, 2017)

En la actualidad el Perú no es ajeno de la inserción de nuevas formas en la comunicación de las personas, es por ello que hoy las personas nos comunicamos empleando las redes sociales. Así éstas son consideradas como el conjunto de personas, comunidades y organizaciones que a diario producen, comparten e intercambian servicios y bienes sociales. (San Martín \& Mujica, 2010). Desde luego que las personas no solo utilizan las redes sociales para comunicarse, sino también lo emplean para el entretenimiento, diversión y mantener las relaciones en línea. Esto permite sostener que las redes sociales hoy constituyen la nueva realidad para comunicarse, entretenerse e informarse.

Las nuevas formas de comunicación que hoy empleamos los humanos, no del todo son ventajosas, también presentan una gran desventaja, ya que la mayoría de la población juvenil lo emplean para diversos fines. En esa línea, el comportamiento de las redes sociales, como el intercambio bidireccional de la información en la sociedad actual trae consigo efectos negativos en los fenómenos económicos, políticos y sociales (Enríquez, 2000) que desde luego ello dirigen el desarrollo en el Perú.

\section{ALFABETIZACIÓN DIGITAL COMO INCLUSIÓN SOCIAL}

En el Perú tal como se puede apreciar en otros Países, una sustancial esperanza de internet no solo se centra en pasatiempos o la enseñanza, sino con mayor énfasis en la cooperación como persona, representante cultural, o integrante de una clase social. Para que estas esperanzas se hagan con efectividad, la gente debe tener bastante instrucción mediática, y estar suficientemente enlazado a organizaciones civiles, no sólo para percibir, sino también para crear contenidos digitales y compartir. La creación de contenidos puede ser comprendida mínimamente el remitir muchos mensajes de correo electrónico, recorrer salas de chat, hasta la invención de una página web, para emplearlos con objetivos cívicos o culturales (Abad, 2016)

En este sentido las TIC como parte vertebral de las sociedades es considerada como el aspecto fundamental que permita la inclusión social y el camino al trabajo, implantando una conexión natural entre inclusión social e inclusión digital; de hecho con muchas variantes en el Perú, indicando como fundamentos primordiales de la brecha digital: brecha digital de género, por motivo de edad, de modelo funcional (discapacidad física o psíquica) por cuestión económica, de índole formativo, de índole geográfico, de tipo ruralurbana y formativo-profesional (García, Leiva, Espíndola, \& Piccoli, 2021) 
La alfabetización digital femenina supone quebrar con la diferencia histórica que existe, no solo por problemas de género, sino también por motivos asociados al despoblamiento y a la necesidad de acceder a las nuevas TIC, tanto para el desarrollo de pesquisas en localidades donde se cambia la pirámide de población, además de ello cada vez se pierden más servicios y oportunidades, que desde luego incrementan las desigualdades y reducen las oportunidades de las personas que proceden en zonas del ámbito rural (García et al., 2021)

La necesidad más importante en el tema de habilidad digital básica, está orientada a la aplicación de las redes sociales encaminadas a la investigación activa de aplicaciones de faenas en equipo. Esto supone un resultado de nivel de desarrollo intermedio en el sector comunicación y competencial del trabajo en equipo, así la inclusión digital no necesariamente implica la inclusión social, por lo que el proceso de alfabetización digital facilita el acceso a las TIC, bridando categorías de soporte y seguimiento social los cuales hacen factible la introducción de los individuos en posición o en peligro de exclusión social. (Travieso \& Planella, 2006)

La utilización de la norma internacional para certificación digital ICDL recomienda que para desarrollar los procesos de alfabetización digital estos deben ser flexibles y no pueden ser aplicados con toda la severidad en los diferentes contextos y ámbitos, dado que los contenidos que son establecidos no concretan sus objetivos para enseñar a personas que están en proceso de utilización de la tecnología. (Solarte, Mario, Urbano, Franco, \& Triviño, 2007)

La integración de las tecnologías en contextos de acción recíproca social propicia en los peruanos, diversos cambios en la forma de comunicarse y de aprendizaje compartiendo información digital. En el sistema educativo actual, el acceso y la aglomeración de la infraestructura digital incita a la necesidad de conceptuar y reconceptuar este proceso, considerando el vocablo alfabetización digital que es una forma de entender y comprender considerando habilidades desarrolladas para participar en el mundo híper-conectado. Considerando la precepción de alfabetización digital, se debe examinar términos como alfabetización en manejo de información, alfabetización mediática y computacional, dichos criterios dirigen las nuevas formas de alfabetización bajo la adhesión de las tecnologías para la educación (George Reyes \& Avello-Martínez, 2021)

\section{NUEVAS FORMAS DE INSERCIÓN EN LA CULTURA DIGITAL}

Hoy en Perú al conmemorar su Bicentenario, se ve invadido por las tecnologías digitales; ya que los peruanos lo tenemos presentes en nuestros hogares, y estos a su vez generaron nuevas dinámicas familiares, causando mucha preocupación en los padres frente a sus hijos con el temor de que cada vez más estén inmersos en el mundo digital (Montoya, Castro, \& Bonilla, 2020). Por otra parte, también ha favorecido a muchas "familias digitales" brindándoles una variedad de oportunidades, en vista de que otras se encontraban con muchas limitaciones.

Aproximadamente hace unos 50 años, los peruanos no fuimos ajenos de ingresar formar parte de la sociedad del conocimiento, estar en las súper carreteras de la 
información, y ello se evidencia en las instituciones educativas, ya que van transformando la visión en el aspecto formador respecto a la investigación y la formación académica de los estudiantes (Flórez et al., 2017). Con el paso del tiempo se ha introducido e insertado en el currículo el uso de las TIC y sobre todo el Internet. Ello ha influenciado principalmente en las relaciones de comunicación entre las personas, así como el acceso abierto y disponible a la gran cantidad de información.

Entre el año 2020 y el presente año, en el Perú y en todos los demás países, se dio por una parte el aislamiento social obligatorio causado por la pandemia Covid-19, y por la otra parte el Ministerio de Educación planteó la continuidad del servicio educativo y no perder el año académico, para ello creó la estrategia "Aprendo en Casa", esto también se dio en el nivel superior; por ello se desarrollaron muchos cursos de capacitación sobre tecnología digital, tanto para estudiantes y docentes. Entonces dicho fenómeno obligó a los profesores a sortear condiciones para adquirir una computadora o laptop, contar con un celular inteligente, conocer y aplicar nuevas tecnologías para desarrollar las clases, emplear el Internet, convertir una habitación en un aula virtual, todo ello pudo ser para los profesores y estudiantes un camino cuesta arriba de principio a fin. (Kalman, 2014)

Los cambios en el sistema de comunicación han cambiado enormemente y en todos los ámbitos, no solo en la educación, estos cambios se vienen dando en la economía, los negocios, la salud, el ocio, la música, el arte y otros rubros, desde luego generando cambios importantes en la sociedad, pero a su vez es una manera de excluir a los que menos tienen (Ramírez, 2014).

\section{ALFABETIZACIÓN DIGITAL PARA UNA PRÁCTICA SOCIOECONÓMICA}

Las generaciones de este siglo, son conocidas como la generación NET, el emplear nuevas tecnologías forman parte de un hábito diario. Se describen por aplicar oportunamente las TIC's, herramientas que permiten impartir e interactuar constantemente con personas próximas y apartadas. A este conjunto de individuos se denominan nativos digitales ya que se comunican fluidamente a través de las mismas. (Arrieta \& Montes, 2011)

El enfoque educativo orientado a la aplicación de las tecnologías de información y comunicación para el incremento de competencias debe apoyarse en una idea colaborativa del aprendizaje. Para ello debe considerar el aprendizaje como un proceso participativo en las destrezas culturales y actividades digitales compartidas por la sociedad, asimismo la intervención y el conocimiento humano se modifican de manera significativa con el anabolismo cultural para difundir el recurso intelectual (Gros \& Contreras, 2006)

El progreso de las Tecnologías en el ámbito de la Información y Comunicación se ha dado con mayor énfasis en el siglo XXI, como una obligatoriedad de una alfabetización en el aspecto digital, así como intergeneracional, producto de la construcción de nuevos ámbitos comunicativos e interactivas más dinámicos entre los ciudadanos. Por otra parte, el estudio del lenguaje científico aproxima la situación de actividades realizadas en la práctica favoreciendo la alfabetización con la familia y la comunidad educativa. La instrucción de identidad del habitante basado en la normatividad de la alfabetización digital, hoy construye la comunidad del conocimiento, cuyo propósito es insertar el 
aspecto digital y la conformación de la sociedad epistemológica (Aguilar-ramos \& Urbano, 2014)

De otro modo, el sistema financiero peruano sale a esplender como un obstáculo para el acceso a las tecnologías y recursos digitales tanto en información y comunicación. La facilidad de ingreso a internet y a equipos informáticos está reducida para un grupo social a nivel mundial. Esta situación el público lo prescribe como un imperativo en las sociedades tecnológicas, donde se considera como una liberación sin equivalencias, convirtiéndose en un privilegio para unas empresas y una debilitación para otras (Ruiz, Suvieres, \& Robles, 2010)

Toda esta brecha digital disminuye si la universidad proporciona a la sociedad los recursos críticos y materiales para usar adecuadamente los recursos digitales. Por lo tanto, la colectividad que abarca el seguimiento, implica ser apreciado donde se permite examinar y colaborar para mejorar los modos de vida, considerando sus dificultades y fortalezas (Gasca, Martínez, \& Tautiva, 2016)

\section{NUEVAS PERSPECTIVAS DE ALFABETIZACIÓN DIGITAL}

Tuvo que transcurrir más de un siglo para que en el Perú se dé la alfabetización digital; la cual hoy se entiende como el dominio que le permita organizar y comprender para luego evaluar, así como generar información empleando la tecnología digital (Rueda Barrios, 2016), pero además del solo uso instrumental, lo que pretende en el ciudadano es desarrollar otras habilidades que posibiliten el fortalecimiento de su capacidad para mantenerse informado, comunicarse con otros y al mismo tiempo movilizarse en el contexto del manejo óptimo de la información.

En la misma línea, la alfabetización digital se entiende como un ejercicio de análisis, la comprensión y el uso de la información disponible al cual se puede acceder mediante los medios digitales (George, 2020). No obstante, el público usuario hoy forma parte de una sociedad interconectada donde la lectura y la escritura digital es un medio para establecer relaciones laborales, educativas y administrativas.

Hoy en día el Perú rumbo a su bicentenario, así como muchos países vecinos en Latinoamérica apuestan cada vez más por el uso de las tecnologías. Así el Ministerio de Educación junto al área de Telecomunicaciones son de mucha importancia y ellos deben garantizar la capacitación y el financiamiento a la educación, brindando el acceso hacia las TIC (Guallo \& Guadalupe, 2018). Desde la década de los ochenta los medios tecnológicos cada vez más nos invaden y en esta era actual se añade lo viral como un interés mediático (Domínguez \& Sánchez, 2020), es por ello que en la actualidad las infografías publicadas en las redes sociales propician la discusión entre los cibernautas, desde luego otros muestran una posición a favor y otro muestran posiciones en contra.

Por otra parte, la alfabetización digital es urgente y necesaria para incrementar la competitividad, así como la productividad de la población, pero hace un siglo eso era imposible, hoy por la coyuntura en la que atraviesa el Perú, así como el sistema educativo peruano debe estar comprometido en brindar facilidades para el logro de las habilidades y competencias digitales ya sea en sus docentes como en estudiantes (González, Olarte, \& Corredor, 2017) 


\section{PERÚ EN EL SIGLO XXI CONSOLIDANDO LA DIGITALIZACIÓN}

\section{El peruano multialfabetizado en el siglo XXI}

El prosumidor del siglo XX soñado por Tofler, en el cual el cassette era de amplio uso, ahora es una aplicación de audio como el "Soundcloud" y aún más como la plataforma de "YouTube" donde se aprende de todo, concordando con la frase "hasta el marido más torpe podría confeccionarse sus propias camisas a medida" (Toffler, 1993)

En siglo XX se pensaba que los aficionados hacia la mecánica estarían en la capacidad de hacer algo más que solo afinar sus automóviles (Toffler, 1993), hoy la realidad nos muestra el cambio sustancial, en el cual se emplean impresoras 3D para la construcción de piezas y las impresiones 4D son lo que se puede imaginar; dado que hoy vivimos en nuevos tiempos en el cual consumidores y productores se cruzan y hasta se fusionan para alcanzar lo que quieren.

Así el concepto de universidad bajo el concepto de multifabetización cambió la manera de entender, es más debemos de comprenderla como un multiverso, complejo, al estilo de Edgar Morín. Es decir, la multiversidad, a partir de la teoría de cuerdas, es la comprensión de diversas formas como la materia se adquiere "multiversos", no existiendo el universo conocido hasta ahora. Este es un nuevo paradigma del saber general, para que exista una nueva multidisciplinariedad es necesario contar con disciplinas que estén abiertas a los fenómenos complejos, con un carácter epistemológico integrador, pues la teoría está condenada a estar incompleta e inacabada (Morin, 1973). La multialfabetización como un aprender complejo, involuntario, que se da en contextos abiertos facilitados por las tecnologías de la información, así como en espacios reales de interacción, superó toda expectativa y hoy surgen nuevos retos no sólo de aprender sino la de gestionar, darle valor agregado, integrar y construir el paradigma complejo como un reto inmediato.

\section{Valores a tomar en cuenta dentro de la alfabetización digital}

Hoy en día mientras transcurre el siglo XXI es común escuchar a los padres, hermanos, tíos, docentes, etc.; es decir, del grupo humano con las cuales nos encontramos rodeados, "que nos encontramos en crisis de valores", esto es frecuente, la realidad trasciende desde que la humanidad atravesó por épocas de grandes cambios en todos los ámbitos, así como, económico, en tecnología, en lo social, incluso cultural. Es por ello, que el buen desempeño de las diversas formas de la competencia dentro de la alfabetización digital implica más que un aprendizaje tecnológico, un factor integral de actitudes, aptitudes, emociones, valores, libertad de expresión y motivaciones (Miranda \& García, 2015). Así la responsabilidad dentro de un grupo de trabajo es imprescindible frente a las acciones para dejar la huella digital y cultural en la inserción de la alfabetización digital.

En dicho contexto, las condiciones fundamentales para un pleno desarrollo y la organización autónoma en el camino a la alfabetización digital se pueden concebir con la aplicación de un sistema equilibrado, así como el empleo de los valores estratégicos, con la relación de los tres ejes que lo compone: la profesionalización, la creatividad y la ética, los cuales se sustentan en la confianza de sí mismo (Pariente, 2006). 
La alfabetización digital es una inclusión de poder tener, impulsar y crear el desenvolvimiento de los valores morales y éticos, tanto a nivel personal y profesional como la responsabilidad, la honestidad, dignidad, sentido de pertenencia, incluso el patriotismo, la responsabilidad social y la empatía (Pérez \& Ferreiro, 2019).

La proyección o la integración en la alfabetización digital es más que teclas y botones, que desde luego exige el uso adecuado de la tecnología y recursos digitales, implicando conocer el para qué, el cómo, el dónde puedo hacer uso de ellos manteniendo la responsabilidad social. Por ello es obvio, el valor de la educación en el pleno fortalecimiento de los valores dentro del seno familiar (Moreno, González, Torres, \& Araya, 2017).

\section{Influencia positiva y negativa del empleo de la tecnología}

Emplear la tecnología resulta sumamente útil y es positivo en la búsqueda de la información usando la "cajita de datos" en el desarrollo de las tareas académicas en los distintos niveles de la formación educacional (Alcibar, Monroy, \& Jiménez, 2018), también permite la interculturalidad, el conocimiento de lenguas extranjeras y más aún el desarrollo de competencias. Por ello en el entorno de la fluidez económica, el empleo de la tecnología se orienta a proponer y efectuar capacitaciones en la inserción del comercio electrónico para mejorar el desarrollo de las pequeñas empresas y fortalecer, así como consolidar las empresas medianas y grandes (Proaño, Lucio, \& Rodríguez, 2020).

La generación de la evolución digital en Perú es perceptible, así la generación Z nació en pleno entorno digital, ya que se cuenta con la presencia de teléfonos móvil, redes sociales, y entre otros. Los dispositivos móviles traen como consecuencia la afección de la comunicación fluida, empleando "emojis" y acrónimos, esta es superior a la generación "Millennials", ya que ellos usaban, por ejemplo: un "Hl" con referencia a "Hola", asimismo un "emoji" para expresar la felicidad o la sonrisa, si bien es cierto que acortan el tiempo de escribir ¿Estamos haciendo lo correcto frente a la enseñanza de los futuros niños? Ello podría considerarse como un aspecto negativo. En la actualidad los nacidos a partir del año 2012 en adelante se denominan la generación Alpha; dicho de otra manera, generación digital, el entorno digital en lo cual se encuentran involucrados antes de cumplir un año son consumidores de videos digitales, capaces de navegar en las aplicaciones del móvil, pc, Tablet, etc. (Instituto de Economia Digital, 2017). El impacto negativo del empleo inadecuado de las diversas modalidades de la tecnología, así como; celulares, internet, consolas, etc. tienen efectos directos en la adicción, afectando el desarrollo integral del niño y favoreciendo el aislamiento social.

Por otra parte, la TIC promueve la igualdad y luchar contra la brecha de género y clase social (Alarcón, 2019), sin embargo, en pleno siglo XXI en el contexto del covid-19 se observa la exclusión laboral, educacional y la desigualdad, por carencia de conocimiento de la empleabilidad de las diversas plataformas y aplicaciones presentes dentro de un hombre multialfabetizado. En síntesis, la tecnología es un medio que aleja de la ignorancia a la humanidad brindándole la información pertinente en los diversos periodos o tiempos, pero a la vez el empleo inadecuado corroe en la inconsciencia. 


\section{Prácticas de inserción de la alfabetización digital}

Con relación a la alfabetización tecnológica, hoy en día se puede observar una gran diferencia entre los estudiantes peruanos actuales y los estudiantes de hace un siglo o más.

De hecho, que los estudiantes actuales muestran más autonomía y son más conocedores de las TIC, esto se da debido a la aparición, así como la inserción de medios tecnológicos en las aulas de los estudiantes. (Cabrales \& Díaz, 2017). Este mismo fenómeno ha ocurrido en la población adulta generando nuevas habilidades cognitivas que fueron evolucionando a instancias hasta desconocidas.

Como principales recomendaciones en procesos formativos de personas mayores dentro del ámbito de las TIC, podemos citar una alfabetización mediática basada en el diseño, un plan de estudios contextualizado para adultos, sensibilización al público respecto a la seguridad en Internet, el valor percibido con los grupos marginados, la autosuficiencia como un componente clave de la alfabetización mediática, asimismo la formación de redes comunitarias, segmentación mediante la composición familiar; es decir quienes tiene nietos, $\mathrm{y}$ finalmente los actores institucionales que deben promover $\mathrm{y}$ motivar el entusiasmo sobre un entorno cambiante frente a medios tecnológicos (Abad, 2016)

Además de lo descrito, la alfabetización digital considerado en la era de la educación virtual "e-learning" como actualmente se conoce, van cambiando y transformando los procesos manuales hacia procesos netamente digitales, también van ampliando estos cada vez más, por lo tanto, la cultura y las formas de aprender o enseñar desde luego se transformaron conforme se insertaban los medios tecnológicos, hasta que hoy en día los medios digitales están subordinando la vida de las personas (Cabrales \& Díaz, 2017)

En la educación virtual es preciso desarrollar nuevas competencias en el aspecto digitales basadas en relación de la sociedad y de forma especial en los ambientes universitarios, por ello Freire y Brunet lo rubricaron manifestando que las nuevas escuelas así como las universidades ya no deben permanecer aislados de esta transformación (Levano-Francia et al., 2019), desde luego estas deben estar guiadas hacia el logro y el desarrollo de las competencias en el rubro digital para así conducir a una sociedad inclusiva y que socialmente se encuentre cohesionada.

En tiempos actuales se exige a todo estudiante desarrollar nuevas capacidades ya sean en el manejo de las redes sociales, Internet, así como los dispositivos electrónicos; de hecho, que es una nueva normalidad, con formas de aprender y enseñar que sean novedosas (Cabrales \& Díaz, 2017)

En la actualidad la aplicación de las tecnologías son bastas, existe todo un universo para aplicarlas en el nivel empresarial y los negocios, insertando hoy en nuestro vocabulario los términos "negocio online" o "e-commerce", desde luego una manera de potenciar las capacidades digitales es contemplar el uso masivo de las nuevas tecnologías tanto para la información como de comunicación para mejorar el desarrollo empresarial en todos los sectores (Arceo Moheno, Ramos Méndez, \& Acosta De la Cruz, 2019)esta 
investigación tuvo como objetivo, mediante un cuestionario cerrado, diagnosticar de manera únicamente descriptiva (sin establecer relaciones entre variables.

En general los peruanos desde hace más de un siglo hasta la actualidad van incrementando el porcentaje de empresas que cuentan con puestos específicos con funciones digitales siendo ello muy ínfimo (Arceo Moheno et al., 2019)esta investigación tuvo como objetivo, mediante un cuestionario cerrado, diagnosticar de manera únicamente descriptiva (sin establecer relaciones entre variables, desde luego que ello se aprecia así debido a la exclusión de las tecnologías, porque en algunos lugares alejados de la sierra así como de la selva peruana aún no llega la ansiada tecnología y si llega, llega con ciertas interferencias o una señal muy baja.

El Perú está dando hoy un gran salto tecnológico, desde luego obligado por la pandemia Covid-19, dado que al encontrarnos en cuarentena y aislados, los peruanos están enfrentándose a distintos factores, que desde luego inciden en la adquisición de bienes y prestación de servicios a través de Internet o empleando medios tecnológicos. Por ello los factores que deben considerar cuando se inicia un negocio "online" son el acceso a una tarjeta de crédito, la experiencia de uso de datos privados y el nivel de confianza de las personas para realizar una compra en sitios online. (Salazar, Mondaca, \& Rodríguez, 2018)

\section{CONCLUSIÓN}

Los sistemas de comunicación con que contaba el peruano del siglo XX ya no son los mismo en el siglo XXI; estos se han innovado y otros fueron reemplazados, en la actualidad utilizamos medios de comunicación digital como celulares inteligentes, el Internet y las redes sociales.

La tecnología digital presenta aspectos positivos y negativos para la sociedad peruana, por una parte, la inclusión y apertura hacia un mundo globalizado, pero para ser parte de ello debemos contar con una alfabetización digital. Por otra parte, la exclusión, ya que excluye a un grupo de la sociedad que aún no cuenta con los medios y recursos necesarios.

Desde el ministerio de educación y las universidades se debe promover en los peruanos la cultura digital y su alfabetización, considerando una educación integral basado en valores que permitan el desarrollo cultural, social, económico y tecnológico en nuestro país.

\section{REFERENCIA BIBLIOGRÁFICA}

Abad, L. (2016). La alfabetización digital como instrumento de e-inclusión de las personas mayores. Prisma Social Revista de Ciencias Sociales, (16), 156-204.

Aguilar-ramos, C., \& Urbano, A. (2014). La necesidad de alfabetización digital e intergeneracional en la familia y la escuela. Revista DIM: Didáctica, Innovación y Multimedia, (28), 1-16.

Alarcón, V. (2019). ¿Cómo influyen las redes sociales en la educación de adolescentes? In Aproximación Periodística Y Educomunicativa Al Fenómeno De Las Redes 
Sociales (pp. 1105-1125). Barcelona, España: McGraw-Hill/Interamericana de España, S.L.

Alcibar, M. F., Monroy, A., \& Jiménez, M. (2018). Impacto y Aprovechamiento de las Tecnologías de la Información y las Comunicaciones en la Educación Superior. Información Tecnológica, 29(5), 101-110. https://doi.org/10.4067/S07180764 2018000500101

Arceo Moheno, G., Ramos Méndez, E., \& Acosta De la Cruz, J. T. (2019). Una visión de las competencias digitales de empresarios de Villahermosa, Tabasco. 1323-1323. Retrieved from http://www.web.facpya.uanl.mx/vinculategica/vinculategica_ 5_2/A.46.pdf

Arrieta, A., \& Montes, D. (2011). Alfabetización digital: uso de las TIC’s más allá de una formación instrumental y una buena infraestructura. Revista Colombiana -RE-CIA, 3(1), 180. https://doi.org/10.24188/recia.v3.n1.2011.360

Ayllón, J. R., Gutiérrez, B., \& González, M. (2008). Filosofía y ciudadanía. Ariel Filosofía, $245 \mathrm{p}$.

Cabrales, O., \& Díaz, V. (2017). El aprendizaje autónomo en los nativos digitales. Conhecimento \& Diversidade, 9(17), 12. https://doi.org/10.18316/rcd.v9i17.3473

Domínguez, A. M., \& Sánchez, S. (2020). Aproximación periodística y educomunicativas al fenómeno de las redes sociales. Madrid, España: McGraw-Hill/Interamericana de España, S.L.

Enríquez, R. (2000). Redes sociales y pobreza: mitos y realidades. Revista de Estudios de Género. La Ventana, 1(11), 36-72. https://doi.org/https://doi.org/10.32870/ lv.v1i11.462

Ferrer, C. (2017). Cura analítica y nuevas formas de comunicación. 1-8.

Flórez, M., Aguilar, A., Hernández, Y., Salazar, J. P., Pinillos, J. A., \& Pérez, C. (2017). Sociedad del conocimiento, las TIC y su influencia en la educación. Espacios, 38(35), 39-50.

Fontana, J. (2005). La historia de los hombres: El siglo XX. Barcelona, España: Crítica.

Fukuyama, F. (1992). El Fin de la Historia y el último hombre. In El estado universal y homogéneo.

García, F. J., Leiva, J. J., Espíndola, E., \& Piccoli, J. F. A. (2021). Inclusión social de mujeres rurales a través de programas de alfabetización digital para el empleo. Revista Complutense de Educación, 32(1), 15-25. https://doi.org/10.5209/rced.67590

Gasca, L. F., Martínez, E., \& Tautiva, J. A. (2016). Comunicación para el cambio social en la emergencia de la alfabetización digital. Revista Nexus Comunicación, (19), 148-167. https://doi.org/10.25100/nc.v0i19.668 
George Reyes, C. E., \& Avello-Martínez, R. (2021). Alfabetización digital en la educación. Revisión sistemática de la producción científica en Scopus. Revista de Educación a Distancia (RED), 21(66), 1-21. https://doi.org/10.6018/red.444751

George, C. (2020). Alfabetización y alfabetización digital. Transdigital, 1, 1-17 González, D., Olarte, F., \& Corredor, J. (2017). La alfabetización tecnológica: De la informática al desarrollo de competencias tecnológicas. Estudios Pedagógicos, 43(1), 193-212. https://doi.org/10.4067/S0718-07052017000100012

Gros, B., \& Contreras, D. (2006). La alfabetización digital y el desarrollo de competen cias ciudadanas. Revista Iberoamericana de Educación, (42), 103-125.

Guallo, J. F., \& Guadalupe, S. E. (2018). La alfabetización digital en Ecuador en el siglo XXI. Atlante: Cuadernos de Educación y Desarrollo, (101), 1-11.

Instituto de Economia Digital. (2017). Las 6 Generaciones de la Era Digital. ICEMD. Retrieved from https://cdn5.icemd.com/app/uploads/2018/12/Estudio_6generaciones-de-la-era-digital-.pdf

Kalman, J. (2014). Más allá de las explicaciones comunes: La incorporación de la tecnología y la cultura digital en el aula. Revista Peruana de Investigación Educativa,

6(6), 33-62. https:// doi.org/10.34236/rpie.v6i6.40

Levano-Francia, L., Sanchez Diaz, S., Guillen-Aparicio, P., Tello-Cabello, S., Herrera-Pai co, N., \& Collantes-Inga, Z. (2019). Digital Competences and Education. Propósitos y Representaciones, 7(2), 569-588. Retrieved from http://www. scielo.org.pe/pdf/pyr/v7n2/a22v7n2.pdf

Miranda, A., \& García, J. (2015). De la alfabetización digital a la alfabetización múltiple. la experiencia de un programa de investigación. 3er. Congreso Internacional de Investigación Educativa. Educación y Globalización, 13.

Montoya, Y. del V., Castro, J. C., \& Bonilla, M. H. (2020). Más allá del control parental: redefiniendo a la familia digital. Anis Do SENID.

Moreno, N. M., González, A. C., Torres, A. C., \& Araya, J. (2017). Alfabetización digital a padres de familia en el uso de las redes sociales. Alteridad. Revista de Educación, 12(1), 8-19. https://doi.org/10.17163/alt.v12n1

Morin, E. (1973). El Paradigma Perdido: Ensayo de Bioantropología. Editorial Kairós, S.A.

Pariente, J. (2006). Los valores y las TICs en las instituciones educativas. Pixel-Bit, Revista de Medios y Educacion, (28), 63-76.

Pérez, Y., \& Ferreiro, J. F. (2019). Los valores en la cultura digital. Revista Caribeña de Ciencias Sociales, (2019-06).

Proaño, W. P., Lucio, A. del J., \& Rodríguez, R. Y. (2020). Accounting under the perspective of electronic devices in micro-enterprise contributions of jipijapa Manabí. Avances, 22(2), 222-238. 
Ramírez, D. (2014). Las nuevas formas de la exclusión digital. En-Claves Del Pensamiento, VIII (15), 85-109.

Rueda Barrios, O. B. (2016). Alfabetización digital y apropiación social: dimensiones que aportan en la construcción de ciudadanía. Puente, 10(1), 73-77. https://doi.org/10.18566/puente.v10n1.a08

Ruiz, S., Suvieres, M. Á., \& Robles, A. (2010). ¿Hacia dónde vamos? La brecha digital y sus consecuencias educativas y socioeconómicas. Jornadas Internacionales Tic, Educación y Sociedad, 1-9.

Salazar, C., Mondaca, C., \& Rodríguez, J. (2018). Comercio electrónico en Chile: ¿qué factores inciden en la decisión de compra? Revista Academia y Negocios, 4(1), 114.

San Martín, L., \& Mujica, S. (2010). Redes Sociales: La nueva forma de comunicación de los jóvenes universitarios. Latin American and Caribbean Conference for Engineering and Technology (LACCEI 2010), 3, 1-10.

Solarte, S., Mario, F., Urbano, O., Franco, A., \& Triviño, G. (2007). B-Learning para Capacitación de Profesores en Alfabetización Digital: Una Estrategia para el Mejoramiento de la Inclusión Social en el Resguardo Indígena de Guambía. Revista Avances En Sistemas e Informática, 4(3), 65-76.

Toffler, A. (1993). La tercera ola. Bantam Books.

Travieso, J. L., \& Planella, J. (2006). La alfabetización digital como factor de inclusión social: una mirada crítica. UOC Papers: Revista Sobre La Sociedad Del Conocimiento, (6), 7. 